\title{
Design of a Four-Element Array Antenna for 5G Cellular Wireless Networks
}

\author{
Dang Thi Tu My \\ Quy Nhon University \\ Binh Dinh, Vietnam \\ dangthitumy@qnu.edu.vn
}

\author{
Tran Thi Huong \\ Huong Ban Mai Education Company Limited \\ Da Nang, Vietnam \\ huonggnouh@gmail.com
}

\author{
Huynh Nguyen Bao Phuong \\ Quy Nhon University \\ Binh Dinh, Vietnam \\ huynhnguyenbaophuong@qnu.edu.vn
}

\author{
Bui Thi Minh Tu \\ Danang University of Science and Technology, \\ The University of Danang, Da Nang, Vietnam \\ btmtu@dut.udn.vn
}

\begin{abstract}
This paper proposes a four-element array planar antenna based on a single antenna that combines the Double Positive (DPS) and Epsilon Negative (ENG) materials. The single antenna consists of a microstrip segment (which is equivalent to a DPS material) connected to a grounded via microstrip segment (which is equivalent to an ENG material). T-Junction power dividers with one-input and two-output ports are used for feeding the two-element and the four-element array antennas. The proposed array antenna is designed to operate optimally at 30GHz frequency with Finite Element Method (FEM)-based simulation. The obtained simulation results show that the proposed array antennas have good radiation performances, in which the four-element array antenna has a $-10 \mathrm{~dB}$ bandwidth ranging from 28.7 to $33.4 \mathrm{GHz}$ and 12.9dBi gain.
\end{abstract}

Keywords- Double Positive (DPS) material; Epsilon Negative (ENG) material; array antennas; zeroth-order resonance; $5 G$ systems

\section{INTRODUCTION}

The rapid development of the millimeter wave communication systems poses many challenges in matching the requirements of these systems, especially the antenna designs for $5 \mathrm{G}$ communication systems [1-2]. These modern antennas are required to be lightweight, to have small size, and to be fabricated easily. The conventional antennas are usually large because the operating wavelength is always relative to their size. Related to this issue, there have been many techniques proposed to reduce the size of the antennas. Transmission Line Metamaterials (TL-MM) [3] are employed to construct small resonant antennas. It is well known that the Composite Right/Left-Handed (CRLH) Transmission Line (TL) has the unique property of an infinite-wavelength wave at a specific non-zero frequency where permittivity and permeability are zero [4-5]. The infinite-wavelength wave property is applied to many microwave components (power dividers, Zeroth-Order Resonators (ZOR), ZOR antennas [6-8], etc.). Nevertheless, the radiation of these antennas is the same with the monopole radiation, whereas wireless imaging transmission systems and space communications require a compact antenna with patchlike radiation [3]. ZOR happens in the Meta-structured Transmission Line (MTL) with ENG [5, 8]. Several models have been proposed to decrease the resonance size of the mushroom-shaped antenna that consists of a rectangular patch with a series gap, is grounded, and has negative permittivity in the specific frequency band [7,9]. In order to improve the size and power of the ZOR antenna, DPS material is added [3]. The antenna pattern is designed on two components with different electromagnetics. By optimizing the DPS segment size, the antenna length can be shortened. With the presence of the DPS, the antenna frequency reduces significantly in comparison with the one zcquired by using only ENG material. To realize a patch-like radiation ZOR antenna, a rectangular patch antenna is loaded with a transversally inhomogeneous substrate [3].

In this paper, a modified CRLH model is used to design a one-dimensional antenna (two-element and four-element) using composite materials. The four-element array antenna exhibits wide-bandwidth and high gain that can be used for the millimeter wave systems at the frequency of $30 \mathrm{GHz}$.

\section{FOUR-ELEMENT ARRAY ANTENNA DESIGN}

The single antenna's design is based on the ENG antenna reported in [4]. T-Junction microstrip power dividers with oneinput and two-input ports (1:2) are used for feeding the fourelement array. This divider requires the power at the input port to be equally divided into two output ports.

\section{A. Single Antenna}

The single antenna consists of a pair of DPS and ENG material MTL. It is designed to resonate at Zeroth-order mode and uses the ENG material which is designed on a mushroom model structure [4]. This mushroom structure is a combination of the rectangular patch with a series gap and a grounded metallic cylindrical pin. It is usually employed to realize the MTL. The MTL with ENG is realized with only the grounded cylindrical pin and has negative permittivity in the specific 
frequency band. To achieve the impedance matching of ZOR antennas, a gap feed $g$ is employed [3]. The antenna is printed on a Teflon substrate with dielectric constant $\varepsilon_{r}=2.1$ and $h=$ $1.57 \mathrm{~mm}$ thickness. They have three cylindrical pins, each with a radius of $0.15 \mathrm{~mm}$. The configuration of the single antenna is shown in Figure 1.

(a)

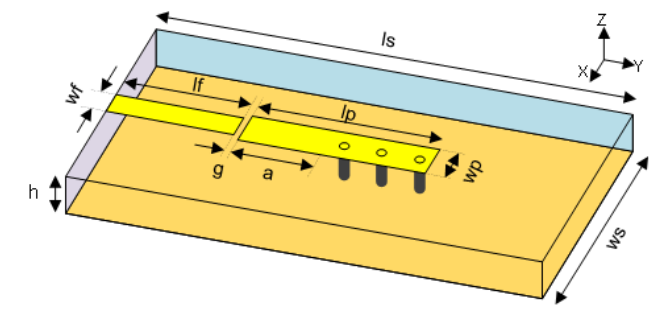

(b)

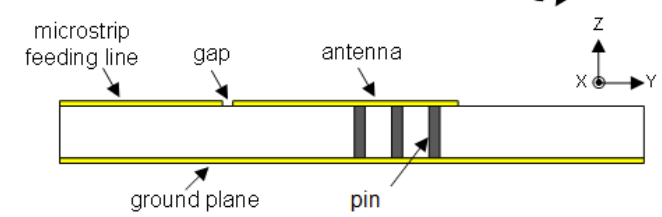

Fig. 1. Configuration of the single antenna: (a) perspective view, (b) side view.

\section{B. Two-element Antenna Array}

A one-dimensional two-element antenna array is effectuated by integrating two single antennas of Figure 1. The T-Junction power divider 1:2 is printed on a Teflon substrate with a relative dieletric constant of 2.1 and a thickness of $1.57 \mathrm{~mm}$. The impedances at the inlet and two-outlet ports of the power divider are $100 \Omega$. The operating frequency is $30 \mathrm{GHz}$. It can be done when (1) is satisfied:

$$
Z_{\text {output } 1}=Z_{\text {output } 2}=Z_{\text {input }}
$$

where $Z_{\text {output } 1}$ and $Z_{\text {output } 2}$ are the impedances of the two outputs and $Z_{\text {input }}$ is the impedance of the input. This divider requires the power at the input to be equally divided into two outputs. In addition, the output signal at the two ports must be in-phase to ensure isolation due to mutual influence. All corners of the divider are cut with an angle of $45^{\circ}$ to reduce reflection. For given characteristic impedance $Z_{0}$ and dielectric constant $\varepsilon_{r}$, the $W / h$ ratio can be calculated as [10]:

$$
\begin{gathered}
\frac{W}{h}=\frac{8 e^{A}}{e^{2 A}-2} \quad \text { for } \frac{W}{h}<2 \\
\frac{W}{h}=\frac{2}{\pi}\left[B-1-\ln (2 B-1)+\frac{\varepsilon_{r}-1}{2 \varepsilon_{r}}\left\{\ln (B-1)+0.39-\frac{0.61}{\varepsilon_{r}}\right\}\right] \\
\text { for } \frac{W}{h}<2 \quad(3) \\
A=\frac{z_{0}}{60} \sqrt{\frac{\varepsilon_{r}+1}{2}}+\frac{\varepsilon_{r}-1}{\varepsilon_{r}+1}\left(0.23+\frac{0.11}{\varepsilon_{r}}\right)
\end{gathered}
$$

Based on (2)-(4), the width of the microstrip line $W$ can be easily calculated with a given height of the substrate $h$. Figure 2 shows the parameters of a one-dimensional two-element array antenna. (a)

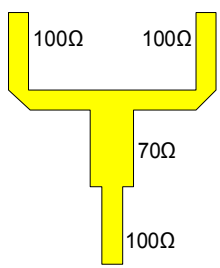

(b)

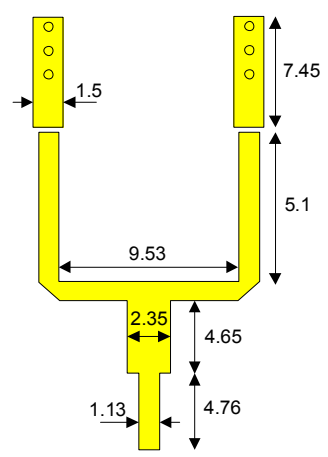

Fig. 2. Two-element antenna array: (a) 1:2 T-Junction power divider, (b) detailed parameters.

\section{Four-element Antenna Array}

Four identical single antennas were integrated with the feeding network to create a higher gain antenna. This feeding network was also built on a Teflon sheet with a thickness of $h=1.57 \mathrm{~mm}$. It consists of three 1:2 T-Junction power dividers that have input and output impedances similar with the one of the two-element array. Each element of the four-element antenna array was excited by the same power and phase (inphase excitation) which are created by the equal power divider. The configuration of the feeding and the four-element array are shown in Figure 3.

(a)

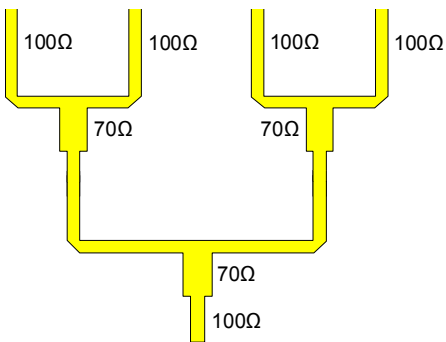

(b)

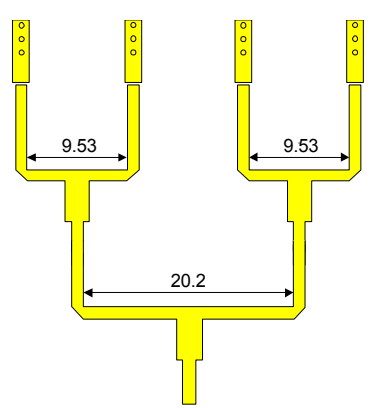

Fig. 3. The configuration of the four-element array: (a) 1:4 T-Junction power divider, (b) detail parameters. 


\section{SIMULATED RESULTS AND DISCUSSION}

The ANSYS Academic Research HF was used for all the simulation cases of the single antenna and the array antennas.

\section{A. Single Antenna}

In this part, the antenna is optimized by investigating the resonant frequency versus different values of the dimension of the antenna's radiator. Firstly, the resonant frequency of the single antenna is verified with various lengths of the DPS segment while the width of the radiator is fixed at $w p=1.5 \mathrm{~mm}$. As can be observed from Figure 4, the center frequency of the antenna decreases when the length $a$ increases. Next, the width $w p$ of the radiator is changed to determine the desired center frequency of the single antenna since the length of DPS $a$ is set as $4 \mathrm{~mm}$. The return loss simulated results of the antenna at the input are shown in Figure 5. It is observed that the center frequency of the single antenna is inversely proportional to the radiator's width. The resonant frequency of the antenna decreases as the length of the radiator increases.

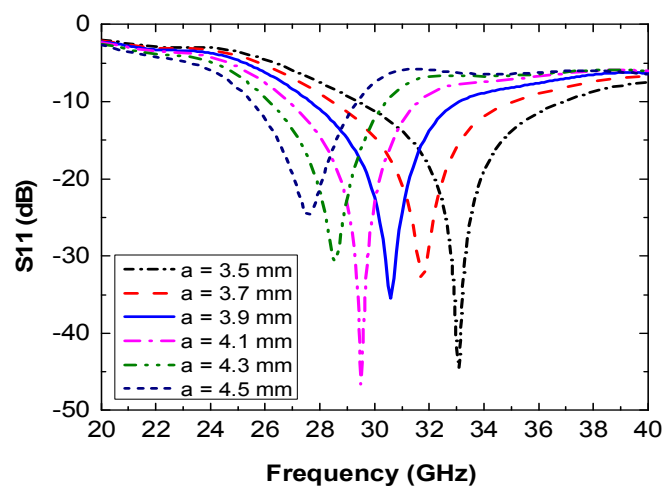

Fig. 4. Reflection coefficient with different DPS length $a$.

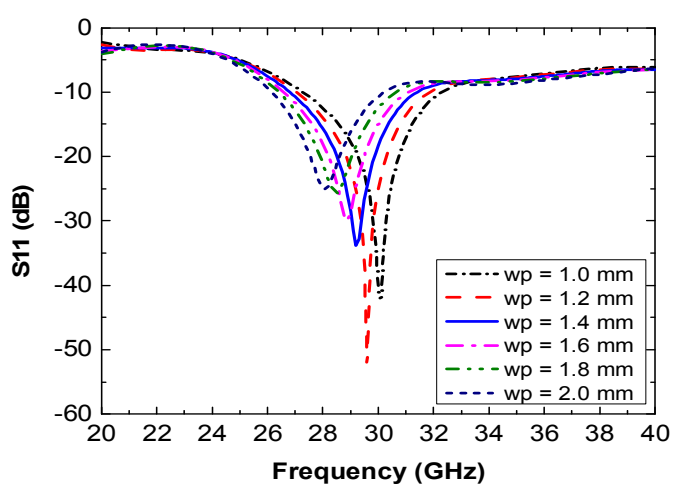

Fig. 5. Reflection coefficient with different antenna width $w p$.

Figure 6 shows the simulated reflection coefficient along with the simulated radiation pattern of the single antenna. Figure 6(a) shows the simulated S11 of the single antenna. It is optimized with the length of DPS $a=3.45 \mathrm{~mm}$ and the radiator width $w p=1.5 \mathrm{~mm}$. The resonant frequency is centered at $30 \mathrm{GHz}$. This can be easily achieved by investigating the length of the DPS segment along with the width of the radiator. The final single antenna has a broad $-10 \mathrm{~dB}$ bandwidth spreading from 27.3 to $32.4 \mathrm{GHz}$. Figure $6(\mathrm{~b})$ presents the simulated radiation pattern of the single antenna. It can be observed that the antenna has an omnidirectional pattern in the $x y$ plane with a gain of $8.0 \mathrm{dBi}$. The optimal dimensions of the single antenna are shown in Table I.

(a)
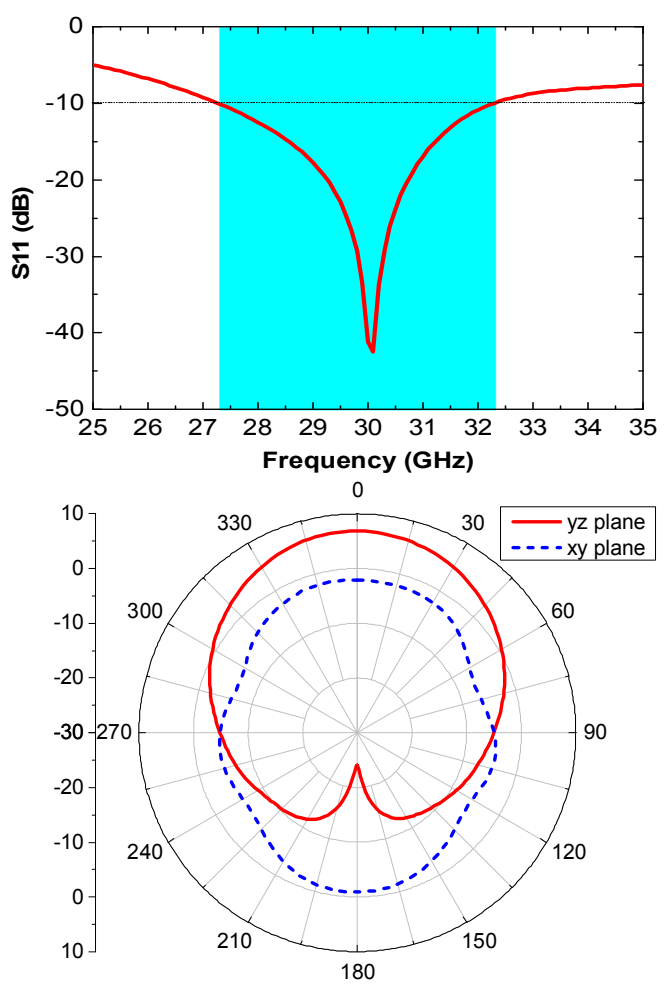

Fig. 6. Simulated S11 and radiation pattern of the single antenna.

TABLE I. OPTIMAL DIMENSIONS OF THE SINGLE ANTENNA (mm)

\begin{tabular}{|c|c|c|c|}
\hline Parameter & Value & Parameter & Value \\
\hline$w s$ & 10 & $w p$ & 1.5 \\
\hline$l s$ & 20 & $l p$ & 7.5 \\
\hline$w f$ & 1.13 & $g$ & 0.2 \\
\hline$l f$ & 5.1 & $a$ & 3.45 \\
\hline
\end{tabular}

\section{B. Two-element Antenna Array}

The simulated S11 of the two-element array antenna is presented in Figure 7(a). It can be seen that the two-element array has $-10 \mathrm{~dB}$ reflection coefficient and bandwidth between $28.4 \mathrm{GHz}$ and $32.6 \mathrm{GHz}$ covering the desired frequency. Figure 7 (b) shows the simulated radiation pattern of the two-element array. The array yields a directional pattern in the $y z$ plane (Eplane) with a maximum gain of $11.0 \mathrm{dBi}$ at $30 \mathrm{GHz}$.

\section{Four-element Antenna Array}

Figure 8(a) presents the simulated S1 1 of the four-element array antenna. The obtained simulation results indicate that the array yields a $-10 \mathrm{~dB}$ bandwidth from $28.7 \mathrm{GHz}$ to $33.4 \mathrm{GHz}$. This array antenna has been successfully designed when its resonant frequency is still centered at $30 \mathrm{GHz}$ as the one of the single antenna. The simulated results of the radiation pattern of the antenna array are shown in Figure 8(b). The antenna exhibits a directional pattern in the E-plane ( $y z$ plane) with a peak gain of $12.9 \mathrm{dBi}$. 


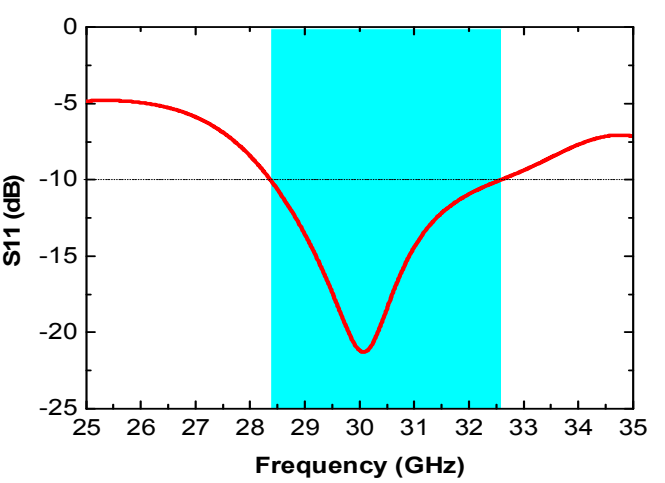

(a)

(b)

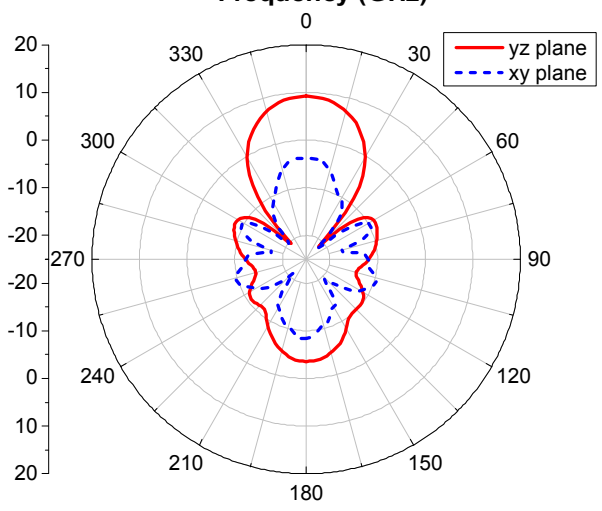

Fig. 7. Simulated S11 and radiation pattern of the two-element antenna array.

(a)
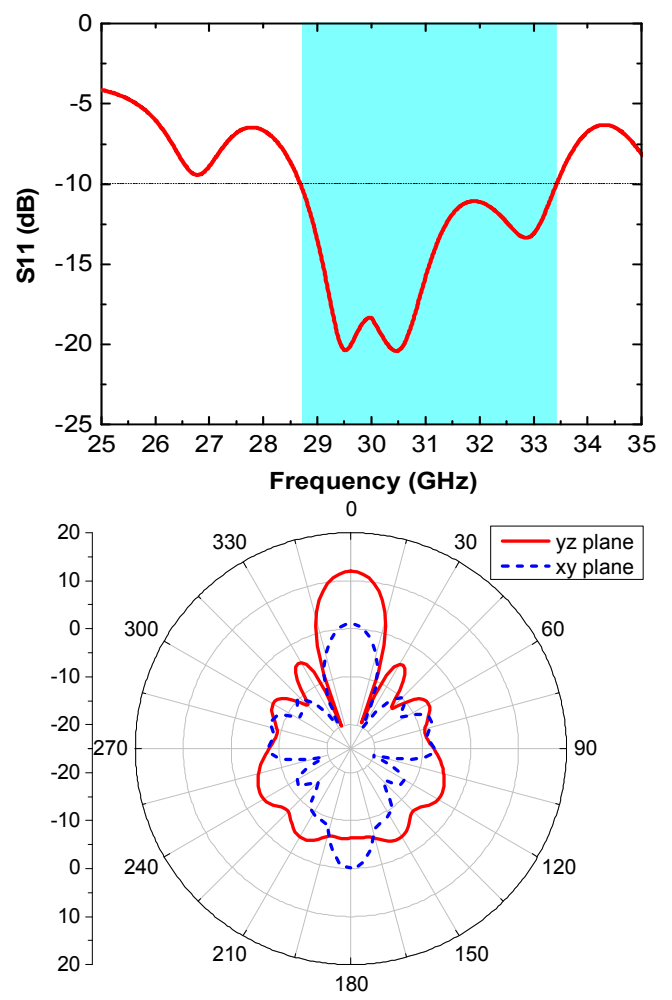

Fig. 8. Simulated S11 and radiation pattern of the four-element antenna Fig. 8 .
array.
TABLE II. COMPARISON BETWEEN THE PROPOSED AND OTHER DIPOLE ANTENNAS

\begin{tabular}{|c|c|c|c|c|}
\hline \multirow{2}{*}{ Ref. } & $\begin{array}{c}\text { No. of array } \\
\text { elements }\end{array}$ & $\begin{array}{c}\text { Feeding } \\
\text { method }\end{array}$ & $\begin{array}{c}\text { Center frequency/ } \\
\text { Bandwidth }(\mathbf{G H z})\end{array}$ & $\begin{array}{c}\text { Gain } \\
\text { (dBi) }\end{array}$ \\
\hline$[\mathbf{1 1}]$ & Array $2 \times 2$ & SIW & $28 / 0.8$ & 9.2 \\
\hline \multirow{2}{*}[\mathbf{12}]{} & Array $2 \times 2$ & Microtrip feed & $28 / \mathrm{NM}$ & 12.3 \\
\cline { 2 - 5 } & Array $1 \times 4$ & Microtrip feed & $28 / \mathrm{NM}$ & 12.5 \\
\hline This work & Array $1 \times 4$ & Microtrip feed & $30 / 4.7$ & 12.9 \\
\hline \multicolumn{4}{|c}{ NM: Not mentioned }
\end{tabular}

A comparison between the proposed 4-element antenna array with related works is shown in Table II. It can be seen that the designed $1 \times 4$ antenna array shows good performance in terms of bandwidth and gain in comparison with the listed array antennas.

\section{CONCLUSION}

In this paper, one-dimensional antenna array has been designed and simulated by combining two material structures that have DPS and ENG properties. Applying array antennas to the design has improved the directional radiation significantly and increased the gain. The simulated results show that the four-element array antenna yields a wide bandwidth spreading from 28.7 to $33.4 \mathrm{GHz}$. In addition, the antenna array represents a maximum gain of $12.9 \mathrm{dBi}$. With this radiation performance, the designed antenna array can be used for millimeter wave applications, especially in the $5 \mathrm{G}$ system.

\section{ACKNOWLEDGEMENT}

This work was conducted at the Calculation and Simulation Laboratory of the Faculty of Engineering and Technology, Quy Nhon University, Vietnam.

\section{REFERENCES}

[1] H. Alsaif, "Extreme Wide Band MIMO Antenna System for Fifth Generation Wireless Systems," Engineering, Technology \& Applied Science Research, vol. 10, no. 2, pp. 5492-5495, Apr. 2020.

[2] M. Azhar and A. Shabbir, "5G Networks: Challenges and Techniques for Energy Efficiency," Engineering, Technology \& Applied Science Research, vol. 8, no. 2, pp. 2864-2868, Apr. 2018.

[3] S. Zhao, Z. Qian, and M. Xiao, "Microwave zeroth-order resonance antenna loaded with a pair of DPS and ENG materials," in 2009 International Conference on Wireless Communications Signal Processing, Nanjing, China, Nov. 2009, doi: 10.1109/WCSP.2009.5371675.

[4] J.-H. Park, Y.-H. Ryu, J.-G. Lee, and J.-H. Lee, "Epsilon Negative Zeroth-Order Resonator Antenna," IEEE Transactions on Antennas and Propagation, vol. 55, no. 12, pp. 3710-3712, Dec. 2007, doi: 10.1109/TAP.2007.910505.

[5] J. H. Park, Y. H. Ryu, J. G. Lee, and J. H. Lee, “A zeroth-order resonator antenna using epsilon negative meta-structured transmission line," in 2007 IEEE Antennas and Propagation Society International Symposium, Honolulu, HI, USA, Jun. 2007, pp. 3480-3483, doi: 10.1109/APS.2007.4396287.

[6] M. A. Antoniades and G. V. Eleftheriades, "A broadband series power divider using zero-degree metamaterial phase-shifting lines," IEEE Microwave and Wireless Components Letters, vol. 15, no. 11, pp. 808810, Nov. 2005, doi: 10.1109/LMWC.2005.859007.

[7] A. Sanada, C. Caloz, and T. Itoh, "Novel zeroth-order resonance in composite right/left- handed transmission line resonators," in 2003 AsiaPacific Microwave Conference, Seoul, Korea, Nov. 2003, vol. 3, pp. 1588-1591. 
[8] J.-G. Lee and J.-H. Lee, "Zeroth Order Resonance Loop Antenna," IEEE Transactions on Antennas and Propagation, vol. 55, no. 3, pp. 994-997, Mar. 2007, doi: 10.1109/TAP.2007.891875.

[9] N. K. Kiem, H. N. B. Phuong, Q. N. Hieu, and D. N. Chien, "A Novel Metamaterial MIMO Antenna with High Isolation for WLAN Applications," International Journal of Antennas and Propagation, vol. 2015, Jul. 2015, Art. No. e851904, doi: 10.1155/2015/851904.

[10] D. M. Pozar, Microwave Engineering, 4th ed. Wiley, 2011.

[11] Y. Feng, B. Yang, Y. Ji, and J. Zhou, "A $28 \mathrm{GHz}$ Millimeter-Wave Antenna Array with SIW Feeding Network," in 2019 International Conference on Microwave and Millimeter Wave Technology (ICMMT), Guangzhou, China, May 2019, doi: 10.1109/ICMMT45702.2019.8992115.

[12] J. Khan, D. A. Sehrai, and U. Ali, "Design of Dual Band 5G Antenna Array with SAR Analysis for Future Mobile Handsets," Journal of Electrical Engineering \& Technology, vol. 14, no. 2, pp. 809-816, Mar. 2019, doi: $10.1007 / \mathrm{s} 42835-018-00059-9$.

\section{AUTHORS PROFILE}

Dang Thi Tu My received the B.E. and M.E. degrees from Hanoi University of Science and Technology in 2000 and 2004 respectively. From 2016 she is a Ph.D. student at the Department of Electronic and Telecommunication Engineering, University of Science and Technology, the University of Danang. From October 2000 to present, she is a lecturer of the Faculty of Engineering and Technology, Quy Nhon University. Research interests: Electronics and Telecommunications, Ultra-high frequency and antenna technology, and Artificial Networks.

Huynh Nguyen Bao Phuong received the B.E, M.Sc. and D.E degrees from Hanoi University of Science and Technology, Vietnam in in 2003, 2007, and 2014 respectively. Now he is a senior lecturer of the Faculty of Engineering and Technology, Quy Nhon University, Vietnam. His research interests are High Impedance Surfaces (HIS), microstrip and metamaterial antennas, and MIMO antennas.

Tran Thi Huong received the B.S degree in Electrical and Electronics Engineering from Utsunomiya University, Japan in 2004 and M.E. and D.E degrees in Electrical and Electronics Engineering from Tokyo Institute of Technology, Japan in 2006 and 2010 respectively. From 2010 to 2019, she was a lecturer at the Department of Electronic and Telecommunication Engineering, The University of Da Nang, University of Science and Technology. Research interests: dielectric resonator, material measurements in millimeter - wave bands, and high frequency planar antennas.

Bui Thi Minh Tu received her $\mathrm{Ph} . \mathrm{D}$. degree from the Universite de Technologie de Compiegne, France in 2010. She started teaching at the Faculty of Electronic and Telecommunication Engineering, The University of Danang, University of Science and Technology in 2010 and since $01 / 2015$, she is the Vice-Dean of the Faculty. Her research interests include communication systems, signal processing, renewable energy, and sustainable development. 